\title{
Corpus delicti. Denken, ein Ort des Verbrechens
}

Arno BöHler, Susanne Valerie Granzer

Lecture-Performance im Rahmen von Philosophy On Stage \#3

26. 11. 2011, Haus Wittgenstein, Wien. ${ }^{1}$

Bühne:

Die Bühne ist durch eine Gazewand vom Zuschauerraum getrennt. Dahinter ein schwarzer Konzertflügel. Von ihrer Mitte sticht ein Steg, wie eine ausgestreckte Zunge, schräg in die Leere des Zuschauerraums. Darauf ein Holzstuhl, eine einfache Glühbirne, am Ende eine Badewanne. Sieben leere Notenpulte in einer Reihe vis á vis.

Akteur_innen:

Die Musik - Die Stimme der Gestimmtheit - oszilliert zwischen Gesetzgeber, Richter, Henker, Helfer und Freund.

Der Philosoph, der Freund Nietzsches - die Stimme der Philosophie als Form EINES BEGEHRENS - auf dem Stuhl, nackt, bis auf ein Badetuch um die Hüften. In der Hand eine Postkarte.

Die ZusCHAUER_INNEN - DIE STIMME DER SympatHiSANT_INNEN ODER MITLÄUFER_ INNEN - links und rechts vom Steg.

Der CHOR - DIE STIMME DER MACHT - sechs Chorist_innen plus Chorführerin. Die CHORFÜHRERIN, anfangs von der Macht gefangen, geht in den Widerstand und verwandelt sich schließlich in - DIE STIMME DER AFFIRMATION, Freundschaft und Liebe, die sich mit der Philosophie paart.

1 Bei den Zitaten in diesem Text handelt es sich um Montagen. Die Abbildungen sind Fotos aus der Lecture-Performance Corpus delicti. Video der Lecture-Performance: http://homepage.univie.ac.at/arno.boehler/php/?p=4417 vom 26. 6. 2013. 
Die Stimme Der Musik. (Live-Musik, 20 sec.)

DER CHOR: »Was haben wir Menschenseelen in irgendeinem schwarzen Abgrund dieses schwarzen Himmels bloß verbrochen, dass wir mit dem Leben bestraft werden? Wie zur Vergeltung einer ungeklärten Schandtat reißt man uns aus einem gestaltlosen, schmerzlosen, namenlosen Frieden und pfercht uns in strampelnde, fressende Körper, die von ihrem Hunger und Durst, ihrem Hass, ihrer Angst oder der nackten Blödheit getrieben, am Ende doch auf irgendeinem Schlachtfeld des Lebens verstümmelt werden. Und selbst wenn es uns gelingt, alt und gebrechlich zu werden, [...] gehen wir nach dem Ratschluss irgendeines gnadenlosen Schöpfers schließlich doch zugrunde - an unserer Lebensgier, an unserem Zerstörungswillen oder zugrunde am bloßen Lauf der Zeit. Und unsere Reste, faulend oder zu grober Asche verbrannt, fallen zurück in die Gestaltlosigkeit, in die Ursuppe irgendeiner nebelhaften Erbschuld, aus der sich dann das sogenannte Dasein [...] noch einmal und immer wieder erheben darf, um auf die immergleiche Art zu enden. «²

Stimme aus dem Off plus Tippen einer Schreibmaschine, Schrift wird auf Gaze projiziert.

Der FReUnd NieTzSCHes (Nietzsche an Overbeck und Köselitz. Stimme aus dem Off): ${ }^{3}$ Ich bin ganz erstaunt, ganz entzückt! Ich habe einen Vorgänger und was für einen! Ich kannte Spinoza fast nicht: dass mich jetzt nach ihm verlangte, war eine >Instinkthandlungr. Nicht nur, dass seine Gesamttendenz gleich der meinen ist - die Erkenntniß zum mächtigsten Affekt zu machen - in fünf Hauptpunkten seiner Lehre finde ich mich wieder [...]: er leugnet die Willensfreiheit -; die Zwecke -; die sittliche Weltordnung -; das Unegoistische -; das Böse -; [...] meine Einsamkeit, die mir [...] oft, oft Athemnoth machte [...], ist wenigstens jetzt eine Zweisamkeit. - Wunderlich! Übrigens ist mein Befinden gar nicht meinen Hoffnungen entsprechend. [...] Schon 6 schwere, zwei- bis dreitägige Anfälle!! [...] Die Intensitäten meines Gefühls machen mich schaudern und lachen - schon ein Paarmal konnte ich das Zimmer nicht verlassen,

2 Christoph Ransmayr: Odysseus, Verbrecher. Schauspiel einer Heimkehr, Frankfurt a.M.: Fischer 2011, S. 11-12.

3 Textcollage aus Friedrich Nietzsche: Sämtliche Briefe, Nr. 135 -136, KSA Bd. 6, 2. Auflage, Berlin/New York: Walter de Gruyter ${ }^{2} 2003$, S. 111-112. 
aus dem lächerlichen Grund, dass meine Augen entzündet waren - wodurch? Ich hatte jedes Mal den Tag vorher auf meinen Wanderungen zuviel geweint, und zwar nicht sentimentale Thränen, sondern Thränen des Jauchzens; - Ach, Freund, mitunter läuft mir die Ahnung durch den Kopf, dass ich eigentlich ein höchst gefährliches Leben lebe, denn ich gehöre zu den Maschinen, welche zerspringen können!

In herzlicher Liebe [F. N]
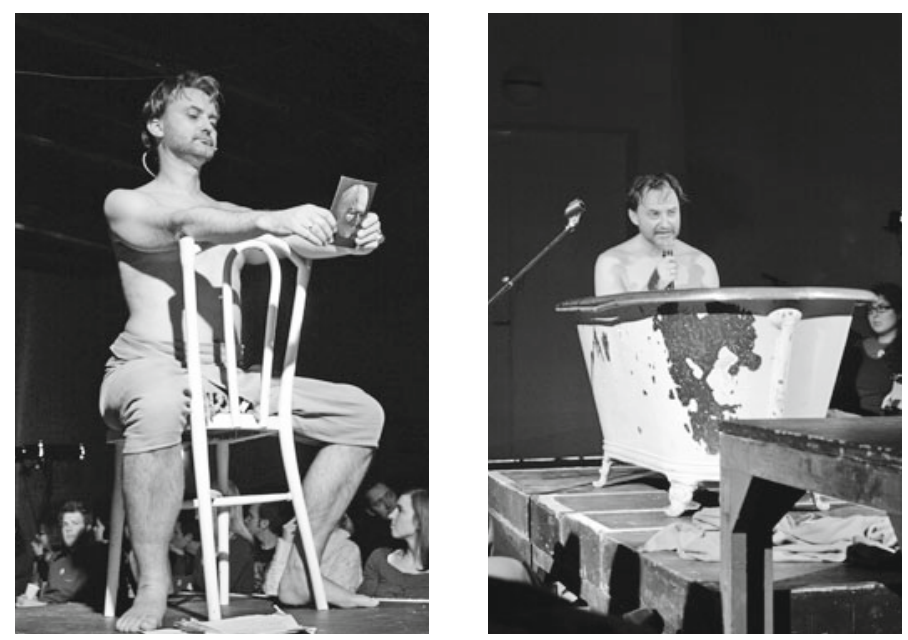

DER FREUND NIETZSCHES (live): Lieber Rätselfreund! Schon sechs schwere Migräneanfälle. - Meine Sorge um Dich ist groß. Aber auch mein Erstaunen darüber, dass Du inmitten solcher leiblicher Torturen Tränenströme vergießt: Nicht des Schmerzes, wie Du mir schreibst, sondern Freudentränen.

Teurer Freund! Du schreibst mir, es wäre eine >Instinkthandlung ‘ gewesen, die Dich gerade jetzt, inmitten Deiner in alle Himmelsrichtungen auseinander berstenden Leiblichkeit, nach ihm verlangt hatte - nach Spinoza. Spinoza und Du. Was für ein denkwürdiges Band kündigt sich hier an? Das Denken zum mächtigsten Affekt machen! Die Erkenntnis zum mächtigsten Affekt machen! Die Philosophie zum mächtigsten Affekt machen! SIE, die Philosophie, ALS Form eines Begehrens lesen lernen - als Prozess eines Geschehens denken lernen, in dem das Begehren beginnt auf sich selbst aufmerksam zu werden selbstreflexiv zu werden. Ich mir. Du dir. Wir uns. Ihr Euch. 
Lieber Freund. Ach! - Was die Menschen alles Liebe nennen? Wer kennt ihn noch, diesen Schauder des Denkens, von dem Du sprichst. Diesen Rausch der Erkenntnis, den die Griechen nicht umsonst Philo-Sophia nannten: Liebe zur Weisheit, Leidenschaft des Denkens. Erkennen, als eine Form von Liebe, als Pathos des Denkens, als Modus des Affizierens und Affiziertwerdens von der Welt! / Zur-Welt-sein, schreibt unser Freund Merleau-Ponty. / Unser zur Weltsein heißt uns zu denken. / Es heißt uns, uns, die wir das Denken lieben, / als eine heiße, sublime Form des Liebens zu denken. / Unser In-der-Welt-sein heißt uns ins Denken zu kommen - / uns vom Denken anfallen zu lassen, / bis unsere Körper ins Denken fallen. / Ihm anheim fallen. / Bis Denken eben der Fall ist. / Bis ihm ein Körper, / dieser, mein Körper hier, verfällt.

Auftritt CHOR plus CHORFÜHRERIN mit überdimensionalen Gesetzbüchern vis à vis durch die Eingangstüre, die in den Zuschauerraum führt. Graue Arbeitsanzüge, Sportschuhe, schwarze, strumpfartige Kopfbedeckungen. Alle legen die riesigen Bücher auf die Notenpulte.

DIE CHORFÜHRERIN: Michel Foucault. Überwachen und Strafen. Kontrolle der Tätigkeiten $\mathrm{I}{ }^{4}$

DER CHOR: Es geht um eine Zwangsbindung der Körper an den herrschenden Produktionsapparat. Die instrumentelle Codierung des Körpers ist das Ziel dieser Tortur. / Die gemessene und bezahlte Zeit muss eine Zeit ohne Fehl und Makel sein, in welcher der Körper ganz seiner Pflichttätigkeit hingegeben ist. / Wie lässt sich die Zeit der Individuen kapitalisieren? Wie lässt sich in jedem von ihnen, in ihren Körpern, ihren Kräften und Fähigkeiten ihre Zeit auf nutzbringende und kontrollierbare Weise kulminieren? Wie lassen sich profitable Dauerhaftigkeiten organisieren? / Durch ununterbrochene Kontrolle und Druck der herrschenden Produktionsverhältnisse - Effizienz, Effizienzsteigerung, Übertragung ökonomischer Organisationsprinzipien auf alle Lebensbereiche, globale Herrschaft der Ökonomie - durch diesen permanenten Zwang wird die Herstellung einer vollständig nutzbaren Zeit zu gewährleisten versucht.

4 Textcollage aus Michel Foucault: Überwachen und Strafen. Die Geburt des Gefängnisses, Frankfurt a.M.: Suhrkamp 1994, S. 193-202. 
DIE CHORFÜHRERIN: Michel Foucault, Überwachen und Strafen, Kontrolle der Tätigkeiten $2:{ }^{5}$

DER CHOR: Im richtigen Einsatz des Körpers, der einen richtigen Einsatz der Zeit erlaubt, darf nichts müßig und nutzlos bleiben. Nur ein disziplinierter, von der Macht geformter, ihr angepasster Körper kann ein leistungsstarker Träger sein, dessen Code den gesamten Körper von der Fußspitze bis zum Zeigefinger erfasst. / Es geht schlichtweg darum, aus der Zeit immer noch mehr nutzbare Kräfte herauszuholen, um an den Punkt zu gelangen, wo die größte Schnelligkeit mit der höchsten Wirksamkeit eins ist. / Mit der Zeit durchdringen und durchsetzen den Körper alle minutiösen Kontrollen der Macht. Ein solcher Organismus wird zum Organ der herrschenden Macht. Er wird ihr Agent. Sichtbarer Ort ihrer Repräsentation. Reproduktion einer die Macht reproduzierenden Macht. / Dadurch wird die von der Macht durchgesetzte Reglementierung der Tätigkeit, ihre Forderung nach Disziplin und Disziplinierung der Körper zugleich das innerste Konstruktionsgesetz ihrer materiellen Manifestation.

DIE CHORFÜHRERIN: Universitätsgesetz $2002 / \int$ I4. Abs. I: ${ }^{6}$

DER CHOR: Das Universitätsgesetz 2002 gebietet zwingend den Aufbau eines Qualitätsmanagements. Im Weiteren bezieht sich $\ \mathrm{I} 4$ auf universitätsexterne und -interne Evaluierung. Neben Effizienz und Effektivität der Leistungserbringung ist auch deren Qualität ein zentraler Gedanke des New Public Managements. Qualitätssicherungssysteme betonen insbesondere die Steuerung und Kontrolle der Qualität. Der internationale Trend geht in Richtung der Frage, ob und wie Qualitätsmanagementsysteme in Universitäten implementiert werden können, und welche Elemente des »Total Quality Managements« relevant sein könnten.

CHORFÜHERIN: Ende des Zitats.

DER CHOR: Total Quality Managements Total Quality Managements Total Quality Managements Total Quality Managements ...

CHOR bleibt mit verschränkten Armen. Wartet ab.

5 Textcollage aus Foucault: Überwachen und Strafen, S. 195-197.

6 Vgl. Heinz Mayer (Hg.): Kommentar zum Universitätsgesetz 2002, 2. Auflage, Wien: Manz 2010, S. 31-33. 
DeR FReUnd NieTZsChes: Das Denken zum mächtigsten Affekt machen? / Die Erkenntnis zum mächtigsten Affekt machen? / Die Philosophie zum mächtigsten Affekt machen? / SIE, die Philosophie, ALS Form eines Begehrens lesen lernen? / Als Prozess eines Geschehens, in dem das Begehren beginnt, auf sich selbst aufmerksam zu werden. - / Selbstreflexiv zu werden. / Ich mir. / Du dir. / Wir uns? / Ihr Euch.

Spinoza UND Du? / Wenn die Zeit angebrochen worden sein wird, in der die Vernunft des Leibes die Torturen von Effizienz und gewissenhafter Evaluation hinter sich gelassen haben wird, EINST, / dann, sagst Du, lieber Friedrich, / wird ein solches begehrlicheres Bild des Denkens nicht mehr gewillt sein, den Akt des Denkens in einer simplen Opposition zu den Affekten zu denken, / sondern mitten darin. / Mitten in diesem Zwischen, / in dem die Affektionen eines Körpers durch einen anderen Körper stattfinden. / Hier, in diesem Zwischen, genau hier, zwischen Dir und mir, / fungieren Körper als Medien füreinander: / Hier geht ein Körper zum anderen hinüber. / Hier geht einer in den anderen über. / Hier breiten sich die Körper selbst über ihre eigenen Körpergrenzen hinaus aus. / Hier, zwischen mir-hier und dir-dort, / exakt hier, wo ich Haut bin, / in diesem konkreten Zwischenraum zwischen uns, / wo ich mehr als Haut bin, / teile ich mich, / teile ich mich mit: den anderen. / Denen, die mit mir da sind. / Hier gehe ich mit einem Bruchteil meiner Masse, einem Bruchteil meiner Energie über mich selbst hinaus. / Hier fliehe ich mich, / hier entferne ich mich von mir selbst. / Hier wende ich mich anderen Körpern zu, / um in ihnen verankert / gegründet zu werden.

Der menschliche Geist, schreibt Spinoza - / Ethik, Lehrsatz 11 und 13, zweiter Teil: Von der Natur und dem Ursprung des Geistes, / der menschliche Geist ist nichts anderes als die Idee eines wirklich existierenden Körpers.

Aber dieser wirklich existierende Körper ist selbst ein in steter Ausdehnung begriffener Körper. / Kein statisches Ding, sondern ein Körper in Bewegung. / Ein Körper, der andere Körper touchiert. / Der nicht an seiner Körpergrenze endet, / der mit anderen Körpern in Berührung ist. / Physis! / Expandierende Kraft. / Ein Weltweitwerden des Körpers! / Das ist es, was die Natur der Körper ausmacht. / Auch das Denken ist ein solcher physio-logischer Kraft-Akt. / Es denkt nicht nur über den Körper nach, / während es denkt, / es macht dabei auch Gebrauch vom eigenen Körper. / Es lässt ihn, den Körper, im Gebrauch des Körpers immer schon mit-denken: / während es denkt, / wenn es denkt, / und solange es denkt. / Immer schon rührt das Denken an einen Körper, / den es berührt - beim Denken. 
Aber dieser, mein Körper, der von mir denkend berührt wird, / ist immer schon ein Körper unter anderen. / Ein Körper, der mit anderen Körpern da ist, / Mein Körper, der mit diesen anderen Körpern da draußen da ist, / die mit mir mit da sind, / hier und jetzt, / mit-da, / hier und jetzt / mit-denkend, / hier und jetzt ...

Der CHOR wird jetzt von einem Kameramann live mitgefilmt und auf beide Seitenwände projiziert. Das heißt, die Stimme der Macht erhebt sich überall allmächtig und sei es als perfekt einverleibter Disziplinierungs-Apparat. Aber gerade jetzt sondert sich die CHORFÜHRERIN ab. Ihr Platz innerhalb der Macht wird zu einer Leerstelle. Sie zieht ein kleines Buch aus der Tasche.

DER CHOR: Michel Foucault. Überwachen und Strafen. Disziplin: ${ }^{7}$

Die normierende Sanktion: Strafbar ist alles, was nicht konform ist. Unter das Strafsystem der Disziplin fällt die Abweichung von der Regel. Deshalb arbeitet im Herzen aller Disziplinarsysteme ein Strafmechanismus, der mit seinen eigenen Gesetzen, Delikten, Sanktionsformen und Gerichtsinstanzen so etwas wie ein Justizprivileg genießt. Mit einer solcherart hierarchisierten und stetigen Überwachung zur Erhöhung der Produktivität wird die Disziplinargewalt ein »integriertes« System, das von innen her mit der Ökonomie und den Zwecken der jeweiligen Institution verbunden ist.

DER CHOR: Universitätsgesetz 2002 / \I3 III. 4 / Wissensbilanz:

Die Wissensbilanz habe nach bestimmten, betriebswirtschaftlichen Kategorien eine Art »Bestandsaufnahme« von Wissen zu geben. »Diese Form der >Bilanz soll der Tatsache Rechnung tragen, dass für Universitäten [...] Wissen ein zentraler Produktionsfaktor ist $\ll^{8}$.

Nicht definiert wird im Gesetz, \I3 III. 6, »was unter intellektuellem Vermögen, Human-, Struktur- und Beziehungskapital sowie Leistungsprozessen mit ihren Outputgrößen und Wirkungen zu verstehen ist. Es handelt sich um betriebswirtschaftliche Begriffe, die hier erstmals auf den universitären Bereich übertragen werden. «?

7 Textcollage aus Foucault: Überwachen und Strafen, S. 230-231.

8 Mayer (Hg.): Kommentar zum Universitätsgesetz 2002, S. 21.

9 Ebd. S. 22. 
Die Stimme der Affirmation: Franz Kafka: Der Prozeß. Die Verhaftung: »)Ach so<, sagte K., « oder wer auch immer, »und nickte, >die Bücher sind wohl Gesetzbücher, und es gehört zu der Art dieses Gerichtswesens, dass man nicht nur unschuldig, sondern auch unwissend verurteilt wird.« ${ }^{10}$

DER CHOR: Foucault. Überwachen und Strafen. Mittel der guten Abrichtung: ${ }^{11}$

Die hierarchisierte, stetige und funktionelle Überwachung beruht in ihrer schleichenden Ausweitung auf den neuen Machtmechanismen, die sie enthält. Sie entwickelt sich so zu einer autonomen und anonymen Gewalt. Denn die hierarchisierte Überwachung der Disziplin beruht zwar auf Individuen, doch wirkt sie wie ein Beziehungsnetz. Die Macht dieses »Netzes« ist keine Sache, die man innehat, kein Eigentum, das man überträgt; sondern eine Maschinerie, die funktioniert. Zwar gibt ihr der pyramidenförmige Aufbau einen »Chef«, aber es ist der Apparat, der »Macht« produziert. Es ist der Apparat, der die Individuen in seinem stetigen Feld verteilt. Das erlaubt es der Disziplinarmacht, absolut indiskret zu sein. Sie liegt immer und überall auf der Lauer.

\section{Die Stimme Der Affirmation: Kafka: Der Prozeß:}

Einzusehen, »dass dieser große Gerichtsorganismus gewissermaßen ewig in Schwebe bleibt und dass man zwar, wenn man auf seinem Platz selbstständig etwas ändert, den Boden unter den Füßen sich wegnimmt und selbst abstürzen kann, während der große Organismus sich selbst für die kleine Störung leicht an anderer Stelle - alles ist doch in Verbindung - Ersatz schafft und unverändert bleibt, wenn er nicht etwa, was sogar wahrscheinlich ist, noch geschlossener, noch aufmerksamer, noch strenger, noch böser wird.«12

DER CHOR: Foucault: ${ }^{13}$ Der perfekte Disziplinarapparat wäre ein vollkommenes Auge, dem nichts entginge - und auf das alle Blicke gerichtet wären. Man müsste die Disziplin zu einer nationalen Sache machen. Ein solcher Staat wird eine dauerhafte und leicht zu führende Administration haben. Diese wird den großen Maschinen gleich sein, die die größten Wirkungen hervorbringen.

Die Stimme der Affirmation: Kafka. Der Prozeß. Der Advokat: ${ }^{14}$

10 Franz Kafka: Der Prozeß, Frankfurt am Main: Fischer 1950, S. 46.

11 Textcollage aus Foucault: Überwachen und Strafen, S. 228

12 Kafka: Der Prozeß, S. 104.

13 Textcollage aus Foucault: Überwachen und Strafen, S. 218-224.

14 Textcollage aus Kafka: Der Prozeß, S. 94-95. 
Sie sind zu unnachgiebig, Josef K., oder wie immer Sie heißen mögen. Gegen dieses Gericht kann man sich nicht wehren, man muss das Geständnis machen. Es gibt keinen Irrtum. Die Behörde wird, wie es im Gesetz heißt, von der Schuld angezogen. Machen Sie doch bei nächster Gelegenheit das Geständnis. - Und wenn ich das Geständnis nicht mache? fragte K. Oder wer auch immer.

\section{CHOR mit Gesetzesbüchern ab.}
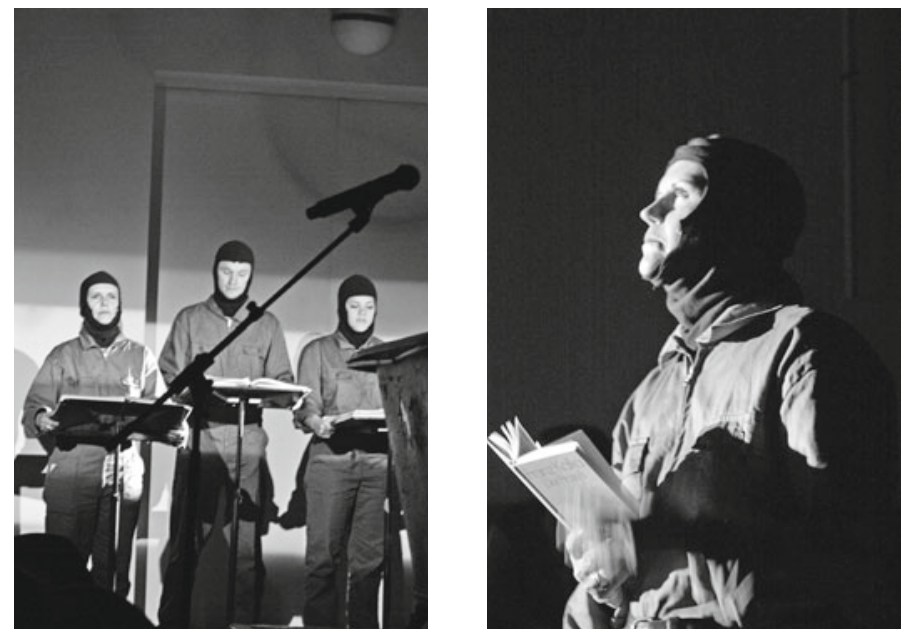

DER FREUND NIETZSCHES: Lieber Friedrich! Vielleicht darf ich Dir an dieser Stelle die Lektüre eines jungen Denkers anempfehlen, dessen Schriften mir sehr wichtig geworden sind: Gilles Deleuze. / Er hat kürzlich ein denkwürdiges Buch über Dich geschrieben - Nietzsche und die Philosophie. / Auch er liebt Spinoza, wie wir beide. / Er hat Deinen Namen explizit neben DIE Philosophie gesetzt - Nietzsche und die Philosophie. Weil Du, wie er meint, Dich in Deinem ganzen Corpus neben sie gesetzt hättest. Neben die klassische Tradition DER Philosophie als Identitätsphilosophie. / Du seiest zwar aus ihr hervorgegangen und in ihr groß geworden, ihr zugleich aber auch entwachsen. Schlussendlich seiest Du sogar ihr radikalster Fremdkörper geworden: / Ihr Untergang und ihr Übergang: / Und daher auch ihr Neuanfang. / Ein konkreter Übergang in etwas Neues, Kommendes! Eine Leidenschaft des Denkens sei durch Dich noch einmal entfacht worden, / fast wie damals, als die Griechen, die guten alten Griechen, der Philosophie den Namen eines Begehrens gegeben haben. / Da- 
mals! Damals, bevor sie zur Wissenschaftslehre wurde, die Philosophie. / Bevor sie Wissenschaftsphilosophie wurde - eine blutleere Metatheorie des Wissens, die jeglichen Bezug zum Philein, / zum Begehren der Erkennenden selbst, / zur Erkenntnis als einer Form des Begehrens, / zur Leidenschaft des Denkens verloren hat. / Und damit auch ihre gesellschaftliche Relevanz.

Was für ein Dreigestirn: Spinoza - Deleuze und Du! / Denken, vollzogen im Medium »Körper«! / In einer dem Körper angemessenen Sprache! / Ist es nicht das, wofür Eure Namen einst gestanden haben werden! / Spinoza, Deleuze und Du? - / EINST! / Denn noch scheint mir dieses Bild des Denkens erst im Kommen zu sein ... / Noch findet dieses Denken nur auf Bühnen statt. / Noch!

\section{Die STIMME DER MUSIK.}

Auftritt Stimme der Affirmation. Jetzt in einem blutroten Arbeitsanzug. Sie liest aus dem Gesetzesbuch.

Die Stimme der Affirmation: 27. Juli i656. Auszug aus dem Gemeindebuch Amsterdam: ${ }^{15}$

Die Herren des Stadtrats tun Ihnen kund, dass sie, nachdem sie die üblen Ansichten und Werke von Baruch de Espinoza seit einiger Zeit kennen, sich auf verschiedene Weisen bemüht haben, ihn von seinen schlechten Wegen abzubringen, und ihn nicht heilen konnten; ganz im Gegenteil erhielten sie täglich schlimmere Nachrichten von abscheulichen Ketzereien, die er begangen und gelehrt hat, und ungeheuerliche Taten, die er getan, wofür es viele glaubhafte Zeugnisse gibt, die alle [...] bestätigt und belegt worden sind; nachdem das alles in Anwesenheit der Herren Rabbiner untersucht war, beschlossen diese, besagten Espinoza mit folgendem Bann zu belegen:

Nach dem Spruch der Engel, nach dem Wort der Heiligen bannen, trennen, verdammen und verfluchen wir Baruch de Espinoza [...] mit allen Flüchen des Himmels, die im Gesetz geschrieben stehen: Verflucht sei er bei Tag, und verflucht sei er bei Nacht, verflucht sei sein Zubettgehen, verflucht sein Aufstehen, verflucht sei er beim Hinausgehen und verflucht beim Eintreten; möge der Herr ihm nicht verzeihen, so dass des Herrn Zorn und seine Eifersucht gegen diesen Menschen entbrennen [...]

15 Bearbeitete Strichversion aus Yirmiyahu Yovel: Spinoza. Das Abenteuer der Immanenz, Göttingen: Steidl 1994, S. 19-20. 
Die Herren des Stadtrats geben bekannt, dass niemand mit Baruch de Espinoza sprechen darf, weder mündlich noch schriftlich, noch ihm einen Gefallen tun darf, noch mit ihm unter einem Dach sich aufhalten darf, noch sich ihm auf vier Ellen nähern darf, noch ein Papier lesen darf, das von ihm gemacht oder geschrieben ist.

DER CHOR (jetzt aus dem Off): 370 v. Chr. Platon. Der Staat. Verbot für die Dichter: ${ }^{16}$

Drittes Buch, 4of: Wir müssen diejenigen überwachen, die schmählich über Tod und Schrecken erzählen, die Furcht erregen und vor der Unterwelt Schaudern machen. Je dichterischer sie dies tun, desto weniger dürfen sie gehört werden. Alles Klagen und Jammern werden wir also abschaffen, Politeia, drittes Buch, 4og. Ebenso alle Lachlust, drittes Buch, 4oh. Denn auch übermäßige Lachlust ist schädlich. Allzu leicht kann sie sich in ihr Gegenteil verkehren. Wir dürfen daher alle Darstellungen von Menschen, die von Gelächter oder von Klagen überwältigt werden, in unserem Staate nicht durchgehen lassen. Oder Erzählungen über Unbeherrschtheit wie drittes Buch, 4oi, dass der Olympier Zeus aus Verlangen nach Liebeslust beim Anblick der Hera dergestalt außer sich gesetzt wird, dass er nicht einmal ins Gemach gehen will, sondern gleich dort auf der Erde sich zu ihr zu gesellen begehrt. In unserem Staate ist untersagt, sowohl toll zu sein als Tolles darzustellen. All dies werden wir zu sagen verbieten. Vielleicht auch noch mehr als dies.

Der Freund Nietzsches: Noch ist dieses Bild des Denkens DER KÖRPER im Kommen, / das ich meine. / Noch spricht es erst im Modus des Versprechens. / Die Erprobung eines Denkens der Körper steht hier auf dem Spiel, / das sich nicht einfach damit begnügt, den Begriff von Körperlichkeit zu denken. / Denn »Körper«, schreibt Jean-Luc Nancy in Corpus zu Recht, / »ist in jeder Sprache das eine Wort zuviel. «17 / Das eine Wort, das notwendigerweise lügt. / Es sagt nie das, was es zu sagen vorgibt. / Denn Körper, das ist keine dumme dumpfe Masse. / Körper, das ist die Öffnung der Existenz für ihr eigenes weltweites Drumherum.

ERSte Film-Einspielung: WutTKe/Pollesch: SChMeiss Dein EGo WeG!

16 Freie Textcollage aus Platon, Politeia, III. Buch, 386a-390a.

17 Vgl. Jean-Luc Nancy: Corpus, Berlin: diaphanes 2003, S. 22. 
DER FREUND NIETZSCHES: Gerade Körper verlangen nicht nach sich selbst, / gerade sie verlangen danach, außer sich zu geraten, / sich in Richtung Außenwelt zu veräußern. / Körper sind draußen. / Sie sind ihrer Natur nach draußen. / Draußen IN der Welt. / Sprich drinnen, „in-der-Welt“. / Draußen in der Welt sind sie drinnen in der Welt, / weil sie naturgemäß von einer Welt umgeben sind. / Jeder Körper ist ein Ding an sich, / aber nur, weil er eine Welt um sich herum an sich selbst herumträgt. / Und exakt darum ist er kein Ding an sich, / sondern Raumkörper: / Ein belebtes Ding, / das von seiner Peripherie angegangen wird, das seine Peripherie etwas angeht: / das ist ein Körper. / Ein verwundbarer Hohlraum: / das ist ein Körper. / Dichte einer Weite: / das ist ein Körper. / Singular plural sein: / das ist ein Körper. / Teil einer mit anderen Körpern geteilten Welt, die berührt wird: / das ist ein Körper. / Form des Mitseins in einer mit anderen Körpern geteilten Welt: / das ist ein Körper.

\section{ZWeite Film-Einspielung: Wuttke/Pollesch: Schmeiss Dein EGo WeG!}

DER FREUND NIETZSCHES: »Psyche ist ausgedehnt, weiß nichts davon «18, schreibt Freud in seiner vorletzten Notiz vor seinem Tod. / Jean-Luc Nancy hat diesen Satz zu einem Mantra seiner eigenen Philosophie erhoben. / Psyche IST Haut. / Psyche, das ist nichts da drinnen - da drinnen in mir. / Sie ist umgekehrt vielmehr das Außer-sich-geraten eines Körpers. / Sie ist exakt das, was ihn, diesen Körper hier, / über sich selbst hinaus geraten lässt; / was ihn, diesen, meinen Körper hier, für seine eigene Peripherie empfänglich, intelligibel, vernehmbar macht. / Sie lichtet einen Körper für sein eigenes Drumherum. / Peri Psyches, Über die Psyche, das ist nicht nur der Titel einer berühmten Schrift von Aristoteles: / Peri Psyches, Über die Psyche handelt von nichts anderem als der Peripherie der Körper. / Von ihrem Drumherum, von ihrem Weltweit-Werden! / Er, der Körper, jeder Körper, auch dieser hier badet in ihrem Licht! Psyche!

Psyche ist ausgedehnt, weiß nichts davon. / Sie hat es vergessen. / Dass sie ein nach außen gestülpter, der Außenwelt zu-gewendeter Körper ist. / Dass sie die Zu-Wendung eines Körpers zur Außenwelt ist. / Dass sie der über einen Körper hinaus andere Körper kontaktierende subtile Teil eines Körpers ist: / Der eigene Körper außer sich. / Der eigene Körper, / insofern er über sich selbst hinaus drängt. / Psyche: Das ist eine in Ausdehnung begriffene Körperlichkeit / ganz wörtlich: res ex-tensa. / Keine Innerlichkeit, sondern die Innigkeit der Körper,

18 Vgl. Nancy: Corpus, S. 23. 
insofern sie in-ein-ander, durch-ein-ander, unter-ein-ander, gegen-ein-ander, für-ein-ander, mit-ein-ander verflochten sind. / Psyche, das ist also der Auszug, der Exodus eines Körpers aus sich selbst heraus.

Auch unsere Emotionen sind da draußen. / Die Privatisierungswelle der Gefühle, / und damit auch ihr Ausschluss aus dem öffentlichen Raum, / hat erst in den letzten beiden Jahrhunderten, / in einem kurzen Wimpernschlag unserer Weltgeschichte stattgefunden. Forget it!

Emotion, das war die längste Zeit so und wird auch wieder einmal so geworden sein, ist die Bewegung der Auferweckung, der Animation, der Stimulation und Belebung eines Körpers, und zwar durch das Interesse eines Körpers an einem anderen Körper.

Aufgespannt zwischen dir und mir sind unsere Körper »innig«, »selig«. / Und diese mediale Spannung da draußen, / diese Tension, / dieser Zug zwischen Dir und mir, / zwischen Ihnen und mir, genau das ist die Seele. / Sie liegt immer schon da, ausgebreitet, vor mir, vor dir, aufgespannt, vor unseren Augen, offenkundig.

Lieber Friedrich! Ist es das, was die NÄHE von Euch beiden ausmacht, Spinoza \& Dir? Dass wir nicht wissen, was die Körper, insofern sie rein ihrer Physis folgen, alles zum Ausdruck bringen könnten? Dass es noch keine Ontologie der Körper gibt, als Orte der Exposition des Seyns? Wissen wir, was ein Körper kann? Das Denken der Körper als Öffnung der Körper, als prima philosophia, als erste Philosophie, die dem Denken sein Gewicht zurückgegeben haben wird?

Ist es nicht dieses Versprechen, das Euch beide eint? Nicht als Werkzeug, als Spielzeug wollt Ihr die Körper, damit sie sprechender werden, durch Euer Denken / damit sie denkender werden / reflektierter / vielstimmiger / dunkler, / komplexer / geheimnisvoller / fühlender, zerbrechlicher / fragiler / subtiler, poröser / durch Euer Denken / denkender / liebender?

Die Stimme DeR Musik. (Live-Musik, 62 sec.)

Auftritt CHOR. Er trägt jetzt ebenfalls blutrote Arbeitsanzüge und spricht den Text auswendig. Die Gesetzbücher sind inwendig wirksam geworden. Die Körper wurden erfolgreich codiert. 
DER CHOR: Kafka: Der Prozeß. Ende: ${ }^{19}$

So kamen sie rasch aus der Stadt hinaus. Ein kleiner Steinbruch, verlassen und öde, lag in der Nähe eines noch ganz städtischen Hauses. Hier machten die Herren halt. Jetzt ließen sie K. los, der stumm wartete, nahmen die Zylinderhüte $\mathrm{ab}$ und wischten sich, während sie sich im Steinbruch umsahen, den Schweiß von der Stirn. Überall lag der Mondschein mit seiner Natürlichkeit und Ruhe, die keinem anderen Licht gegeben ist. Nach Austausch einiger Höflichkeiten ging der eine zu K. und zog ihm den Rock, die Weste und schließlich das Hemd aus. K. fröstelte unwillkürlich, worauf ihm der Herr einen beruhigenden Schlag auf den Rücken gab, während der andere Herr den Steinbruch nach irgendeiner passenden Stelle absuchte. Als er sie gefunden hatte, winkte er, und der andere Herr geleitete K. hin. Es war nahe der Bruchwand, es lag dort ein losgebrochener Stein. Die Herren setzten K. auf die Erde nieder, lehnten ihn an den Stein und betteten seinen Kopf obenauf. Dann öffnete der eine Herr seinen Gehrock und nahm aus einer Scheide ein langes, dünnes Fleischermesser, hielt es hoch und prüfte die Schärfe im Licht. K. wusste jetzt genau, dass es seine Pflicht gewesen wäre, das Messer selbst zu fassen und sich einzubohren. Aber er tat es nicht. Alle Arbeit den Behörden nicht abnehmen. Seine Blicke fielen auf das letzte Stockwerk des an den Steinbruch angrenzenden Hauses. Wie ein Licht aufzuckt, so fuhren die Fensterflügel eines Fensters dort auseinander, ein Mensch, schwach und dünn in der Ferne und Höhe, beugte sich weit vor und streckte die Arme noch weiter aus. Wer war es? Ein Freund? Einer, der teilnahm? Einer, der helfen wollte? War noch Hilfe? Gab es Einwände, die man vergessen hatte? Wo war der Richter, den er nie gesehen hatte? Wo war das hohe Gericht, bis zu dem er nie gekommen war? Er hob die Hände und spreizte alle Finger. Aber an K.s Gurgel legten sich die Hände des einen Herrn, während der andere das Messer ihm tief ins Herz stieß und zweimal dort drehte. Mit brechenden Augen sah noch K., wie die Herren, nahe vor seinem Gesicht, Wange an Wange aneinandergelehnt, die Entscheidung überwachten.

Dunkel. Abgang CHOR.

Dritte Film-Einspielung: Wuttke/Pollesch: SChmeiss Dein EGo Weg!

Die Stimme der Musik. (Live-Musik, 65 sec.)

19 Bearbeitete Strichfassung aus Kafka: Der Prozeß, S. 193-194. 
Auftritt Stimme der AfFirmation oben auf der Bühne hinter der Gazewand. Noch im roten Arbeitsanzug. Sie hält eine Stabmaske Nietzsches vor das Gesicht. Die Musik mischt sich vorsichtig in den Text.

Die Stimme Der Affirmation: Friedrich Nietzsche. Das Nachtlied:

»Nacht ist es: nun reden lauter alle springenden Brunnen. Und auch meine Seele ist ein springender Brunnen. / Nacht ist es: nun erst erwachen alle Lieder der Liebenden. Und auch meine Seele ist das Lied eines Liebenden. / Ein Ungestilltes, Unstillbares ist in mir; das will laut werden. Eine Begierde nach Liebe ist in mir, die redet selber die Sprache der Liebe [...] / Nacht ist es: nun bricht wie ein Born aus mir mein Verlangen, - nach Rede verlangt mich. / Nacht ist es: nun reden lauter alle springenden Brunnen. Und auch meine Seele ist ein springender Brunnen. / Nacht ist es: nun erst erwachen alle Lieder der Liebenden. Und auch meine Seele ist das Lied eines Liebenden. «20

»Oh Mensch! Gieb Acht! / Was spricht die tiefe Mitternacht? / >Ich schlief, ich schlief -, / Aus tiefem Traum bin ich erwacht: - / Die Welt ist tief, / Und tiefer als der Tag gedacht. / Tief ist ihr Weh -, / Lust - tiefer noch als Herze-
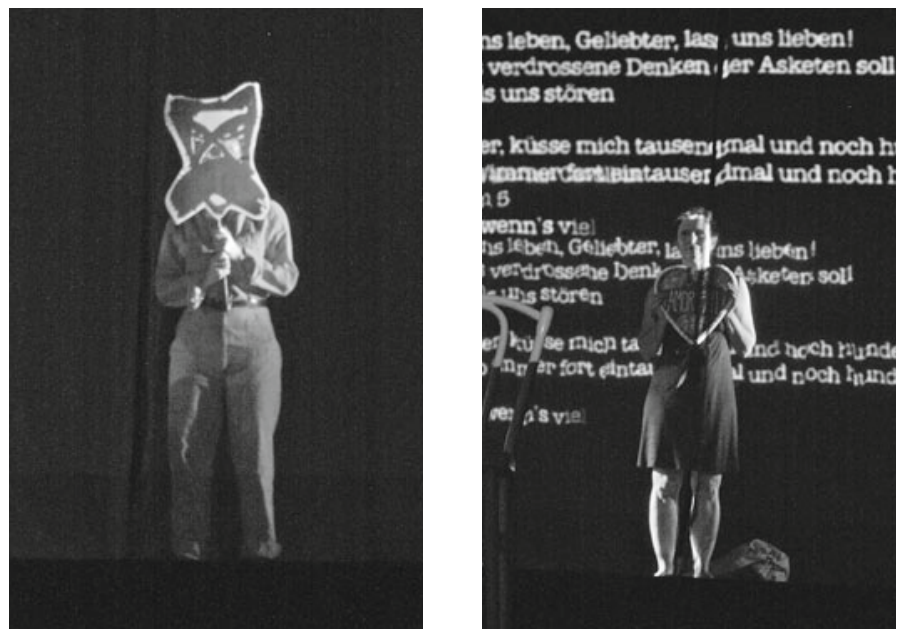

20 Friedrich Nietzsche: Also sprach Zarathustra, KSA Bd. 4, München/Berlin/New York: DTV 1980, S. 136-138. 
leid: / Weh spricht: Vergeh! / Doch alle Lust will Ewigkeit -, / - will tiefe, tiefe Ewigkeit!« 21

Die Stimme Der Affirmation legt die Stabmaske Nietzsches zu Boden, zieht Schuhe und Arbeitsanzug aus. Darunter trägt sie ein kurzes Trägerhemd. Sie nimmt ein riesiges rotes Lebkuchenherz aus dem offenen Flügel, auf dem in Zuckerguss Amor fati geschrieben steht. Es wird dunkler. Das Tippen einer Schreibmaschine ist zu hören und ein Liebesgedicht Catulls, das, wie der Text der Postkarte an Overbeck zu Beginn, auf die Gazewand projiziert wird.

Die Stimme DeR LIeBE: Gaius Valerius Catullus. Carmen 5:22

Lass uns leben, Geliebter, lass uns lieben! / All das verdrossene Denken der Asketen soll / niemals uns stören! // Liebster, küsse mich tausendmal und noch hundert / und so immer fort eintausendmal und noch hundert. / Dann wenn's viele Tausende sind, dann verwirren / wir sie, alle die Küsse, die unzählbaren, / dass kein Neider das Glück uns mindere, wissend, / wie viel süßeste Küsse wir zärtlich uns schenkten.

Nach Ende des Textes tritt die affirmative Stimme DeR Liebe durch den Schlitz in der Gazewand auf den Steg, geht zu dem Philosophen, dem FreUnd NieTzSCHES und steigt nackt zu ihm in die Badewanne. Dabei ist es ganz dunkel geworden. Ein jäher Lichtstrahl auf den Schlitz der Gazewand. Man sieht eine riesige Nietzschemaske, die von einer Figur in blutrotem Arbeitsanzug gehalten wird. Die Stimme der Musik kreischt dissonant in höhnischem Lachen auf, gleichzeitig wird an den unsichtbaren Fäden der Nietzschemaske gezogen, sodass der überdimensionale Schnurrbart Nietzsches wie bei einem Hampelmann, von unten nach oben schnellt - und Nietzsche plötzlich mit auflacht.

Die Stimme der Musik À la Grosses Bollywood Finale. (Live-Musik, 55 sec.)

Philosoph: Arno Böhler, Chorführerin: Susanne Valerie Granzer, Chor: Studierende des Max Reinhardt Seminars, Musik: Wolfgang Mitterer, Bühne: Hans Hoffer

21 Ebd. S. 404.

22 Freie Textcollage aus: Catull-Tibull-Properz. Römische Liebeslyrik, München: Goldmann 1959, S. 11. 


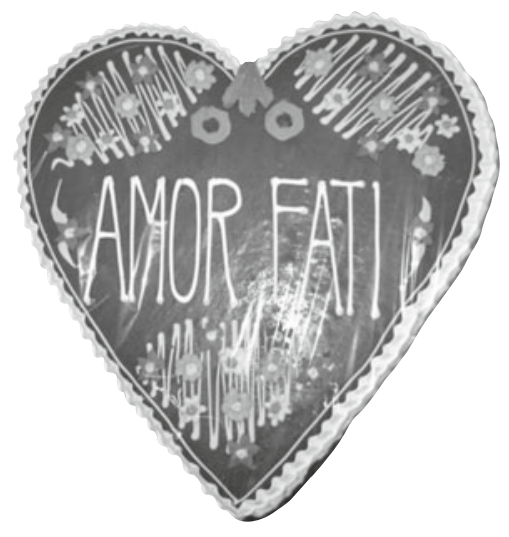

